Modelling the high temperature oxidation of titanium alloys:

\title{
review of analytical models
}

and development of a new numerical tool PyTiOx

C. Ciszak ${ }^{a, *}$, D. Monceau ${ }^{a}$ and C. Desgranges ${ }^{b}$

${ }^{a}$ CIRIMAT, Université de Toulouse, CNRS, INP-ENSIACET, 4 allée Emile Monso - BP 44362, 31030 Toulouse - France

b Safran-Tech, Materials and Processes Department, Rue des Jeunes Bois, Châteaufort, 78114 Magny-les-Hameaux, France

$\underline{\text { Abstract }}$

In order to limit the ecological impact of air traffic and its operating costs, the aeronautical industry is looking for improving engines efficiencies and substitutes to high density Ni-based superalloys. Thus, a wider use of Ti-alloys operating at higher solid-state diffusion laws can be found in the litterature. They are fairly accurate in most cases, but they reveal some intrinsic limitations in specific cases such as temperature transients or thin components. Numerical models were later developed to break down these limitations. First results from a new numerical tool called "PyTiOx" (still under development are presented here. They confirm the intrinsic limitations of analytical models. In the case of thin samples, the numerical model predicts an increase of scaling

\section{Introduction}

Titanium alloys are required to operate at higher temperatures to limit the ecological impact of air traffic and to decrease operating costs. This gives access to better engines efficiencies while challenging heavier alloys, such as Ni-based superalloys. Therefore, ductility, which can lead to structural failure. In addition, this $\mathrm{O}$ dissolution increases the mechanical strength and elastic moduli. Thus, it appears crucial to predict as accurately as possible the high temperature oxidation behavior of Ti-based components in order to improve their sizing and durability prediction.

At high temperature, the oxidation of $\mathrm{Ti}$-alloys is known to be controlled by the diffusion of $\mathrm{O}$ within both the $\mathrm{TiO}_{2}$ and $\mathrm{Ti}$ metallic phases [1]. Thus, $\mathrm{O}$ profiles in the system (oxide and metallic matrix) can be simulated using classical kinetic laws of solid state diffusion [2]. Based on the analytical resolution of the Fick's laws, several analytical models were proposed in the past to predict the shape of diffusion profiles within materials. They range from simple erf function accounting of the diffusion in a single phase EKINOX code was specially developed for the high temperature oxidation of Zr alloys [4,5].

The present work is dedicated to the evaluation of these different analytical models and to the development of a new numerical tool dedicated to the oxidation of Ti alloys at high temperature

\section{Review of common analytical models for diffusion in solids} During Ti alloy oxidation, O dissolution depths are observed to reach about 10 times the associated oxide scale thickness. Consequently, the metal /oxide interface recession can be often neglected when calculating the oxygen diffusion in the metallic matrix.
The O dissolution and inward diffusion in the matrix is the only phenomenon which is considered for predictive calculations. Within this framework, the simplest approach for predicting the O diffusion profile within the Ti matrix, consists in using a simple erf function (Eq. 1) [2] (model 1)

$\left.C_{T \mathrm{~V}}(x, t)=\operatorname{erfc}\left(\frac{x}{2 \sqrt{D_{O}\left(T_{i}\right)}}\right)\left(C_{T \mathrm{i} \mid T \mathrm{Ti}}-C_{0}\right)\right)+C_{0}$

where $x$ represents represents the distance from the metal|oxyde interface (in m), $t$ the time of oxidation (in s), ${ }_{O}(T)$ ) the $\mathrm{O}$ diffusion coefficient within the Ti matrix (in $\left.\mathrm{m}^{2} . \mathrm{s}^{-1}\right), C_{T \mathrm{i}}$ the local $\mathrm{O}$ concentration (in at. fraction) and $C_{T i \mid T i O_{2}}$ and $C_{0 \mathrm{Othe}} \mathrm{O}$ concentrations (in at. fraction) at the $\mathrm{Ti}_{\mathrm{TiO}}$ interface and the initial $\mathrm{O}$ content respectively.

However, the growth of the oxide scale and $\mathrm{O}$ dissolution are actually two correlated phenomena. Therefore, a more complete form of the previous analytical model is necessary, and the solution proposed by Wagner [6] and compiled by Crank [3] for phase (5) within the metallic matrix. The constant $\gamma$ only depends on the system physic-chemical parameters (boundary conditions and diffusion coefficients).

$C_{T_{\mathrm{iO}}}(x, t)=C_{s}-\operatorname{erf}\left(\frac{x}{2 \sqrt{D_{O}\left(T_{\imath} O_{2}\right)^{t}}}\right) \frac{\left(C_{S}-C_{\left.T i O_{2} \mid T_{i}\right)}\right.}{\operatorname{erfc(\gamma )}}$ Eq. 2

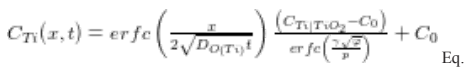

$\varphi=\frac{D_{\left.O, T_{i} O_{2}\right)}}{D_{O}\left(T_{i}\right)} \mathrm{Eq} \cdot 4$

$\xi(t)=2 \gamma{\sqrt{D_{O\left(T i O_{2}\right)}}}^{E q q .5}$

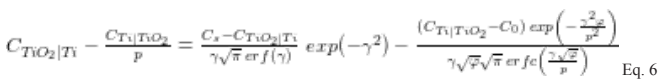

Here, $D_{\mathrm{O}}\left(\mathrm{TiO}_{2}\right)$ represents the diffusion coefficient of oxygen in the oxide scale (in $\left.\mathrm{m}^{2} \mathrm{~s}^{-1}\right), \varphi$ the ratio of the diffusion coefficients of $\mathrm{O}$ in $\mathrm{TiO}_{2}$ and in the Ti matrix (Eq. 4), $C_{S}$ and $C_{T i O_{2}} \mid T \mathrm{~T}$ the $\mathrm{O}$ content (in at. fraction) in the oxide at the oxide|atmosphere and at the oxide|metal interfaces respectively,, the oxide scale thickness (in $\mathrm{m}$ ), $p$ the Pilling and Bedworth ratio and $\gamma$ the coupling constant.

However, in the case of thin samples and very long oxidation treatments, the center of the sample tends to fill up with $\mathrm{O}$ over time up to saturation. This particular behavior is not reproduced by the two previous models. Thus, Pawel [4] proposed a semianalytical model allowing accounting of the finite dimension of specimens (model 3). The new equation describing the O diffusion profile in the Ti matrix is then presented in Eq. 7 .

$C_{T i}(x, t)=\frac{\left.\left[1-\frac{1}{2} \theta(x)\right)\right]}{\left.\left[1-\frac{1}{2} \theta(\xi)\right)\right]}\left(C_{T i \mid T i O_{2}}-C_{0}\right)+C_{0}$ Eq. 7

where $\theta$ is a function of $x$ or $\xi$ presented in Eq. 8:

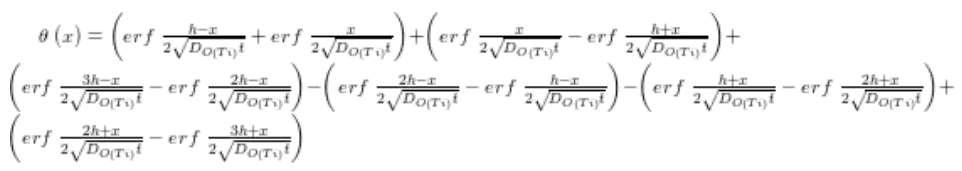

Here, $h$ represents the original thickness of the specimen (in $\mathrm{m}$ )

Figure 1 presents experimental data [7] compared with the results provided by these different analytical models. These experimental data were obtained by EPMA measurements on a Ti $6242 \mathrm{~S}$ alloy oxidized $1000 \mathrm{~h}$ in a $80 \%$ Ar $/ 20 \% \mathrm{O}_{2}$ gas mixture, excluding the formation of nitrides, which are known to influence the overall oxidation kinetic.

(C) The Authors, published by EDP Sciences. This is an open access article distributed under the terms of the Creative Commons Attribution License 4.0 (http://creativecommons.org/licenses/by/4.0/). 


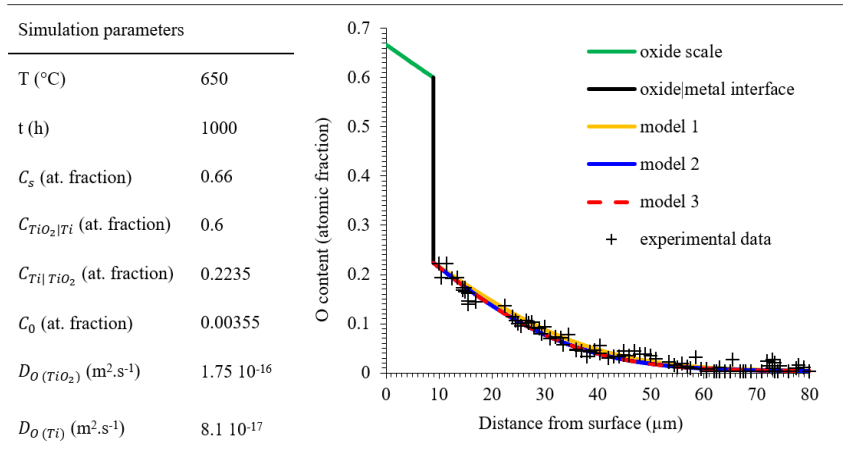

Figure 1: $O$ profiles obtained from analytical models compared with experimental data from [7].

According to these results, the $\mathrm{O}$ profile obtained from the first model appears to be slightly over-valued compared with those obtained from the two other more complete models.

In the particular case of $\mathrm{Ti}$, as for the other elements of the IV $\mathrm{V}^{\text {th }}$ periodic table column, the $\mathrm{O}$ dissolved within the metallic matrix (up to 33 at.\% and 8 at.\% in $\alpha$-Ti and $\beta$-Ti respectively [8]) represents an important contribution to the overall weight gain classically measured during high temperature oxidation experiments. Thus, compared to the simplest model (model 1), model 2 and model 3 allow the direct comparison of calculations with experimental kinetics in terms of weight gain. Furthermore, they also allow following the contribution of each medium (oxide and metal) to the overall weight gain.

By considering the coupling between oxide scaling and diffusion within the metallic matrix, models 2 and 3 are also able to account for the evolution of the oxide scaling kinetics as a function of the initial $\mathrm{O}$ content in the metallic matrix. Results of calculations performed on model 3 with two initial $\mathrm{O}$ contents of 0 and 2 at. $\%$ are presented in Figure 2 .

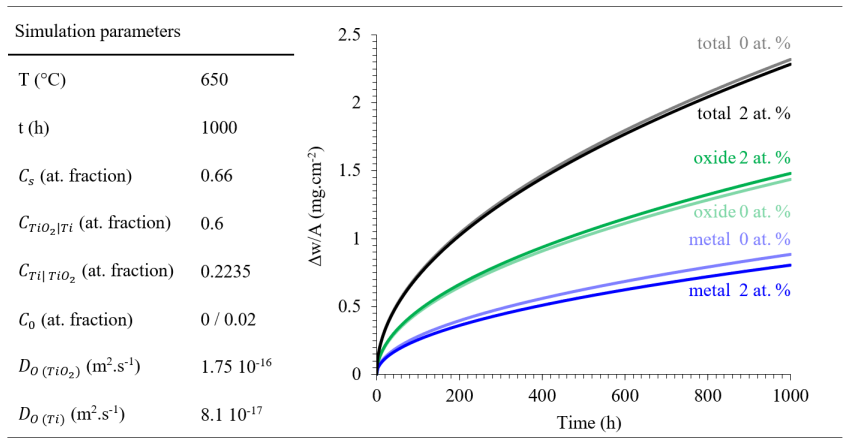

Figure 2: Evolution of calculated weight gains with time for two different $O$ initial contents in the material.

According to the evolution of the weight gain curves presented in Figure 2, it is confirmed that none of both metal and oxide contributions to the overall weight gain can be neglected in this case. This result particularly highlights the advantage of using a complete model which considers diffusion in both oxide and metal.

It also appears that a material containing a larger initial level of $\mathrm{O}$ in the metal will present a lower final weight gain. Moreover, higher oxide scaling kinetics is observed for a sample containing a higher initial oxygen content. This is due to the lower $\mathrm{O}$ quantity teed in this case to form the oxide, when compared to 0 - fee netallic matrix.

Nevertheless, if model 3 accounts of the evolution of the $O$ content at the sample center over time, it does not take into account the influence of such an evolution on the value of the $\gamma$ parameter (according to Eq. 6). Thus, despite the variation of the $O$ content at the sample center over time, no evolution of both diffusion kinetics and oxide scaling kinetics is considered by such analytical models. In the case of thin samples, which can be subjected to saturation of their metallic matrix in $\mathrm{O}$, none of these analytical models supports the impact of a progressive saturation in $\mathrm{O}$ of the metallic matrix on the oxide scaling kinetics (Figure 3).

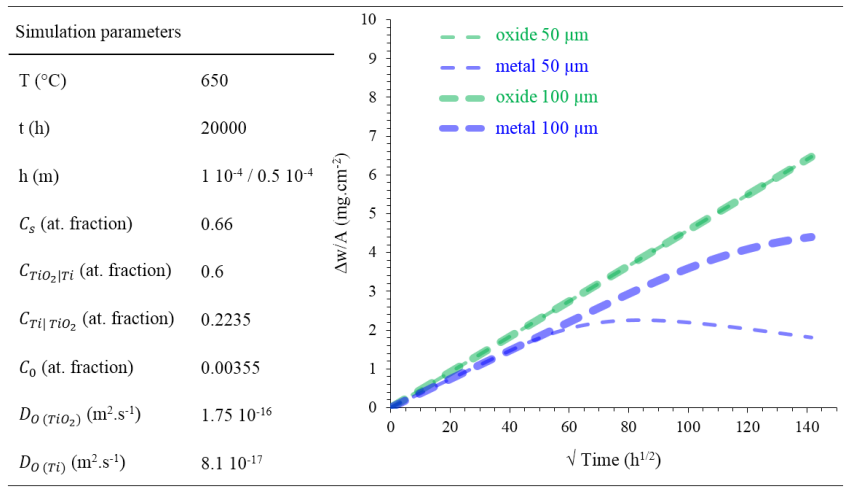

Figure 3: Evolution of calculated weight gains with square root of time for two different sample sizes.

According to Figure 3, only a decrease of the weight gain associated to the metallic part of the sample can be observed, which is attributed to the consumption of this metallic part by the oxide growth. This decrease begins when the weight gain kinetics of the metal start to be balanced by the kinetics of its consumption.

Thus, it clearly appears that any analytical model, even the most complete one, suffers from limitations concerning the potential time and space dependences of its parameters. For instance, accounting for temperature transients, local evolution of diffusion coefficients, local evolution of the volume fractions of phases, redistribution of the alloying elements are such key parameters that should be supported.

Development of a numerical tool for high temperature oxidation of Ti alloys: PyTiOx

In order to break down limitations intrinsic to analytical models, PyTiOx, a new numerical tool was developed, following the approach used for zirconium alloys oxidation [4,5]. It is based on the explicit finite differences numerical scheme applied to a one (its of crystalline lattice. Displacement of the metaloxide boundary is supported and achieved through growth, thinning, creation and deletion of slices in each medium, on both sides of the interface. Initial $O$ profiles are calculated in each medium using model 2 , corresponding to those that would be obtained for the formation of the very thin initial oxide scale thickness. Boundary conditions, i.e. $C_{S}, C_{T i} O_{2} \mid T i_{1}$ and $C_{T i} \mid T i_{2}$ are set to imposed values. They can change with time, but in the following calculations they
were taken constant. At each new time step, oxygen fluxes are calculated between slides using the concentrations calculated at the previous time step. Then, $\mathrm{O}$ concentrations in each slide of both oxide and metal are calculated from matter preservation principles.

A first calculation was performed with the same input data used for calculation presented in Figure 3. The results of the analytical and numerical models are compared in Figure 4. 


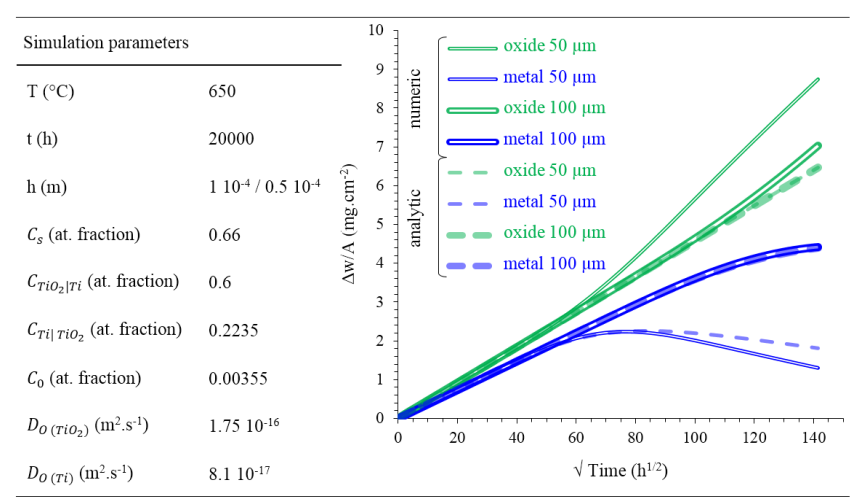

Figure 4: Comparison of evolution of numerically and analytically calculated weight gains with square root of time for two different sample sizes.

According to Figure 4, results from numerical model appears to deviate from analytical model results at around $50 \mathrm{~h}$ and $100 \mathrm{~h}$, for a sample of $50 \mu \mathrm{m}$ and $100 \mu \mathrm{m}$ thick respectively. This occurs when the metal become saturated in $\mathrm{O}$, evidencing a kinetic transition. Indeed, on the contrary of analytical models, numerical model accounts for the feedback over time of the $\mathrm{O}$ content evolution at the center of sample ( $C_{0}$ ) on the oxide scaling kinetics. Thus, in case of thin samples, scaling kinetics follow two successive regimes. The first one, effective at short times of oxidation, will be limited by diffusion of $\mathrm{O}$ in both oxide and metal. The second one becomes effective once metal is saturated in $\mathrm{O}$. At this stage, $\mathrm{O}$ fluxes in metal will tend to decrease and

\section{Conclusion}

A review of the most common analytical models used to simulate the high temperature oxidation of alloys able to dissolve large amounts of $O$, such as Ti alloys, was presented. It has revealed that increase of oxidation kinetics with the initial amount of $O$ in the material can be predicted by models supporting diffusion in oxide and metal with the displacement of the corresponding interface. It has also been shown that metal saturation in $\mathrm{O}$, in case of thin samples, can be predicted by the same kind of model. However, example when using additive manufacturing.

\section{$\underline{\text { References }}$}

[1] P. Kofstad, High temperature corrosion, Elsevier Applied Science, London and New York, 1988.

[2] J. Crank, The mathematics of diffusion, $2^{\text {nd }}$ ed, Oxford, 1975.

[3] R.E. Pawel, Diffusion in a finite system with a moving boundary, J. Nucl. Mater. 49 (1974) 281-290.

[4] B. Mazères, C. Desgranges, C. Toffolon-Masclet, D. Monceau, Modeling Two- and Three-Stage Oxygen Tracer Experiments during High-Temperature Oxidation of Metals with a High Oxygen Solubility, Oxid. Met. 89 (2018) 517-529. [5] B. Mazères, C. Desgranges, C. Toffolon-Masclet, D. Monceau, Experimental study and numerical simulation of high temperature (1100-1250 ${ }^{\circ}$ ) oxidation of prior-oxidized zirconium alloy, Corros. Sci. 103 (2016) $10-19$.

[6] C. Wagner, W. Jost, Diffusion in solids, liquids, gases, New York, 1952

[7] C. Dupressoire et al., Corr. Sci. (2019) (submitted).

[8] H. Okamoto, O-Ti (Oxygen-Titanium), J. Phase Equilibria Diffus. 32 (2011) 473. 